\title{
Study on Reconstruction of Heat Flux in Moving Boundary
}

\author{
Zhijian Wu \\ Institute of Meteorology and Oceangraphy \\ PLA University of Science Technology \\ Nanjing, China, 02580830262 \\ xueyan006@163.com \\ Xueyan Zhang \\ Institute of Meteorology and Oceangraphy \\ PLA University of Science Technology \\ Nanjing, China
}

\author{
Jing Feng \\ Institute of Meteorology and Oceangraphy \\ PLA University of Science Technology \\ Nanjing, China
}

Aixia Zhou

Institute of Meteorology and Oceangraphy PLA University of Science Technology

Nanjing, China

\begin{abstract}
The initial boundary value problem for parabolic equation with Neumann boundary condition is a kind of classical problem in partial differential equations. In this paper,we consider a heat inverse problem with moving boundary. By introducing a density function for potential method, thisproblem is essentially converted into solving an integral equation of the first kind with respect to the density function, which can be solved by the Tikhonov regularizing method. Model function method is used to determine the regularizing parameter. Numerical results are presented to support our inversion schemes.
\end{abstract}

Keywords- heat equation;potential scheme; heat flux; regularization;numerics;

\section{INTRODUCTION}

The initial boundary value problem for parabolic equation with Neumann boundary condition is a kind of classical problem in partial differential equations. The direct problem is to solve the temperature field from given initial and boundary conditions. If the boundary conditions are not specified completely, but with some additional condition given at the interior point of the domain, then it formulates an inverse problem for reconstructing the boundary heat flux, which is illposed. In general, the classical solution to this problem may not exist, also the solution may not depend continuously on the input data. So some regularizing method should be applied to get a stable approximate solution.

The study of system with moving boundary is extensively used in conservancy[2],numerical analysis[4,6], physics[7],water biology[10], construction in national defence[3,5],etc.

\section{THE QUESTION}

On $\Omega:=\{(x, t): x \in(X(t), \infty), t>0\}$,consider the following heat conduction problem with Neumann boundary condition and the initial temperature field

This work was supported in part by ZBYX under Grant Nos. 407010401 and XYJCLL under Grant Nos. KYQYZL001222 .

$$
\begin{cases}\frac{\partial u}{\partial}-a \frac{\partial^{2} u}{\partial x^{2}}=0 & 0 \leq X(t) \leq x \leq+\infty, t \in(0, t) \\ \left.\lambda_{0} \frac{\partial u}{\partial x}\right|_{x=X(t)}+q(t)=0,\left.\frac{\partial u}{\partial x}\right|_{x=+\infty}=0 & t \in[0, t] \\ u(x, 0)=\varphi(x) & X(0) \leq x<+\infty\end{cases}
$$

Where ${ }^{\lambda_{0}}$ are known and $q(t)$ means heat flux.From the Physical point of view,the boundary movement can be conditioned by ablation,thermal shrinkage or linear expansion of the body material.

About the problem described by (1),it is a direct problem to get temperature $u(x, t)$,if $X(t), \varphi(x)$ are known already.However,in fact, the heat flux $q(t)$ is not easy to get,but the temperature on somewhere can be abtained easily.So it is feasible to get $q(t)$ using the metrical data $u(x, t)$.Then it is an inverse problem. Once the $q(t)$ is available ,the temperature $u(x, t)$ can also be confirmed by direct problem.

The inverse problem is to identify $q(t)$ in $[0, \mathrm{~T}]$ from the following additional condition

$$
u(d, t)=f(t), \quad t \in[0, t]
$$

with $d>\max _{[0, T]} X(t)$.However,the real $f(t)$ is difficult to get, the geodesic $f^{\delta}(t)$ is more reasonable,with condition of

$$
\left\|f^{\delta}-f\right\|_{L^{2}[0, T]} \leq \delta
$$


So the inverse problem is convertd to identify $q(t)$ from the geodesic $f^{\delta}(t)$.It will be a Volterra integral equation of the first kind,which is seriously ill-posed,and regularizing scheme is necessary.

\section{CONVERTION OF THE INITIAL CONDITION}

Here,potential method was used to solve the inverse problem,decribed by (1)-(2).So the initial condition $u(x, 0)=0$ is necessary.Assume that the initial condition satisfied the following condition:

$\varphi(x) \in C([X(0),+\infty)), \varphi(X(0))=0, \lim _{x \rightarrow+\infty} \varphi(x)=0$

Let

$$
\psi(x)= \begin{cases}\varphi(x) & x \in[X(0), \infty) \\ 0 & x \in(-\infty, X(0))\end{cases}
$$

And define

$$
Z(x, t)=\int_{-\infty}^{+\infty} \psi(\eta) \frac{e^{-\frac{(x-\eta)^{2}}{4 \alpha t}}}{\sqrt{4 \pi \alpha t}} d \eta
$$

It is easy to prove that $Z(x, t)$ is the solution of heat problem of Cauchy ,with the initial condition $Z(x, 0)=\psi(x)$

lemma 2.1. The new function $v(x, t)$ satisfies

$$
\begin{cases}\frac{\partial v}{\partial t}-\alpha \frac{\partial^{2} v}{\partial x^{2}}=0 & 0 \leq X(t)<x<\infty, t \in(0, T) \\ \left.\lambda_{0} \frac{\partial v}{\partial x}\right|_{x=X(t)}+q(t)+\left.\lambda_{0} \frac{\partial Z}{\partial x}\right|_{x=X(t)}=0 \\ \left.\frac{\partial v}{\partial x}\right|_{x=+\infty}=0 & t \in[0, T] \\ v(x, 0)=0 & X(0)<x<\infty\end{cases}
$$

Where $v(x, t)=u(x, t)-Z(x, t)$. Now

$$
v(d, t)=f(t)-Z(d, t):=f^{*}(t), t \in[0, T]
$$

Then the inverse problem convert to reconstruct $q(t)_{\text {from the }}$ information about $v(d, t)$, where $Z(x, t), X(t)$ and $f^{*}(t)$ are all known.Notice that the $\left.\frac{\partial v}{\partial x}\right|_{x=x(t)}$ should be taken for $\left.\frac{\partial v}{\partial x}\right|_{x=x(t)+}$.Introduce the density function representation for $v(x, t)$ :

$$
v(x, t)=\int_{0}^{t} \frac{\alpha e^{-\frac{(d-X(\xi))^{2}}{4 \alpha(t-\xi)}}}{2 \sqrt{\pi \alpha(t-\xi)}} \omega(\xi) d \xi, \quad X(t)<x<\infty, \quad t \in[0, T]
$$

with $\omega(t) \in C[0, T]_{\text {Using(4), we get that }} \omega(t)$ meets

$$
v(d, t)=\int_{0}^{t} \frac{\alpha e^{-\frac{(d-X(\xi))^{2}}{4 \alpha(t-\xi)}}}{2 \sqrt{\pi \alpha(t-\xi)}} \omega(\xi) d \xi=f^{*}(t), \quad t \in[0, T]
$$

also considering the geodesic data $f^{* \delta}$, with

$$
\left\|f^{* \delta}-f^{*}\right\|_{L^{2}[0, T]} \leq \delta
$$

The (7) is an integral equation of the first kind with respect to the density function $\omega(t)$.Regularizing scheme should be applied to solve this equation to get the approximate solution $\omega^{\partial}(t)$.

Theorem 2.2. If $f^{*}(t)$ is the exact number,then the expression (7) has the unique solution $\omega(t)$ lying in $\mathrm{C}[0, \mathrm{~T}]$.

Proof: The expression(7) is a linear about $\omega(t)$,so it is only to prove that $\omega(t)=0$ when $f^{*}(t)=0$.

Let $f^{*}(t)=0$,so the expression (7) converts to

$$
\int_{0}^{t} \frac{\alpha e^{-\frac{(d-X(\xi))^{2}}{4 \alpha(t-\xi)}}}{2 \sqrt{\pi \alpha(t-\xi)}} \omega(\xi) d \xi=0, t \in[0, T]
$$

$$
K(t-\xi)= \begin{cases}\frac{\alpha e^{-\frac{(d-X(\xi))^{2}}{4 \alpha(t-\xi)}}}{2 \sqrt{\pi \alpha(t-\xi)}} & 0 \leq \xi<t \leq T \\ 0 & \xi \in[t,+\infty) \cup(-\infty, 0)\end{cases}
$$

So $\int_{-\infty}^{+\infty} K(t-\xi) \omega(\xi) d \xi=0$

The left of the equation above is just the convolution of $K(\cdot)$ and $\omega(\cdot)$.Use Fourier Transform on both side of the equation above $\mathscr{F}(K)(\cdot) \mathscr{F}(\omega)(\cdot)=0$

$$
\begin{aligned}
& \text { Because that } \quad K(t-\xi) \neq 0 \text {,so } \\
& \mathscr{F}(K)(\cdot) \neq 0 \text {,then } \mathscr{F}(\omega)(\cdot)=0 \Rightarrow \omega(t) \equiv 0 \text {,proof over. }
\end{aligned}
$$


When use regularization method on expression(7), the approximate solution $\omega^{\alpha}(t)$ of $\omega(t)$ can be get.Then the jump relationship of the potential theory on on boundary $X(t): t \in[0, T]$ can be used to get $q(t)$.The jump relationship[1] is described as below

lemma 2.3. On $X(t): t \in[0, T], v(x, t)$ has the following jump relation

$$
\left.\frac{\partial v}{\partial x}\right|_{x=X(t)+}=\left.\frac{\partial v}{\partial x}\right|_{x=X(t)}-\frac{1}{2} w(t)
$$

Using this Lemma and the boundary condition in (4), $q(t)$ can be solved from

$$
\begin{aligned}
q(t)= & -\left.\lambda_{0} \frac{\partial Z}{\partial x}\right|_{x=X(t)}+\frac{\lambda_{0}}{2}[w(t)+ \\
& \left.\int_{0}^{t} \frac{e^{-\frac{(X(t)-X(\xi))^{2}}{4 a(t-\xi)}}}{\pi^{\frac{1}{2}} a^{\frac{1}{2}}(t-\xi)^{\frac{3}{2}}} \frac{X(t)-X(\xi)}{2} w(\xi) d \xi\right]
\end{aligned}
$$

where ${ }^{\omega(t)}$ has been determined approximately from (7) by regularizing scheme.

\section{NUMERICAL EXAMPLE}

In this section,we will present a numerical example to show the validity of our algorithm.Consider the following heat problem

$$
\begin{cases}\frac{\partial v}{\partial t}-\alpha \frac{\partial^{2} v}{\partial x^{2}}=0 & 0 \leq X(t)<x<\infty, t \in(0, T) \\ \left.\lambda_{0} \frac{\partial v}{\partial x}\right|_{x=t}+q(t)+\left.\lambda_{0} \frac{\partial Z}{\partial x}\right|_{x=X(t)}=0 & t \in[0, T] \\ \left.\frac{\partial v}{\partial x}\right|_{x=+\infty}=0 & X(0)<x<\infty \\ v(x, 0)=0 & \end{cases}
$$

Where $\varphi(x)= \begin{cases}\sin x & x \in[0,2 \pi] \\ 0 & \text { else } \quad, X(t)=t\end{cases}$ So

$$
K \omega(t)=\int_{0}^{t} \frac{\alpha e^{-\frac{(x-t)^{2}}{4 \alpha(t-\xi)}}}{2 \sqrt{\pi \alpha(t-\xi)}} \omega(\xi) d \xi
$$

and

$$
K^{*} \varphi(\xi)=\int_{\xi}^{T} \frac{\alpha e^{-\frac{(x-t)^{2}}{4 \alpha(t-\xi)}}}{2 \sqrt{\pi \alpha(t-\xi)}} \varphi(t) d t
$$

Let

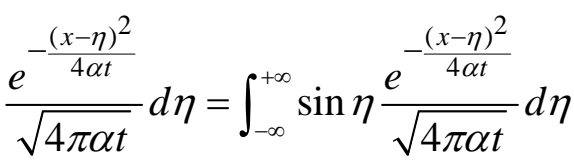

and

$v(x, t)=u(x, t)-Z(x, t)$,the inverse problem is just converted to get $\omega(t)$ from the equation below

$$
v(d, t)=f(t)-Z(d, t)=\int_{0}^{t} \frac{\alpha e^{-\frac{d^{2}}{4 \alpha(t-\xi)}}}{2 \sqrt{\pi \alpha(t-\xi)}} \omega(\xi) d \xi:=f^{*}(t)
$$

If $\alpha=1.0, d=2.0, \pi=3.14159, T=1.0$,the $f^{*}(t), \quad \omega(t)$ and $q(t)$ can be given by the artificial boundary.Just shown as Figure1
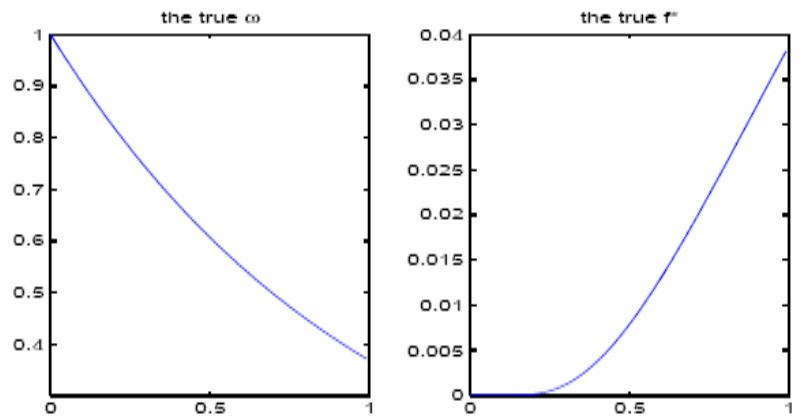

Figure 1. the real $\omega(t)$ and $f^{*}(t)$

Let $f^{\delta}=f^{*}(t)+\delta \times$ unifrnd $(-1,1, N, 1) f^{*}(t)$,where unifrnd $(-1,1, N, 1)$ can give the rand array between $[-1,1]$ of $N$-dimension .Here $N=100$.

Fig2 is the figure of $\omega(t)$ used by the prior selection of regularization parameter.We can see that when regularization parameter $\alpha$ and error $\delta$ is in balance,The geodesic and the real is alike.

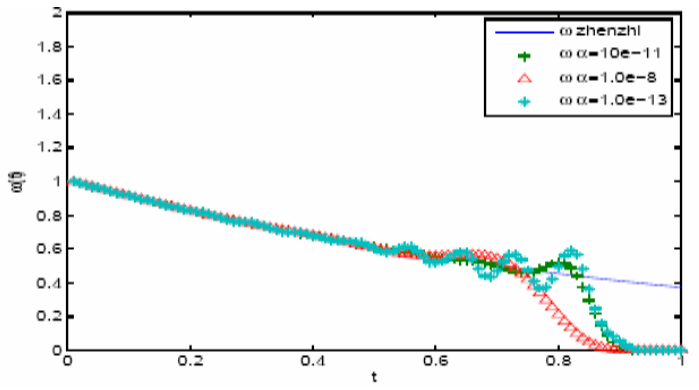

Figure 2. figure of $\omega(t)$ when $\delta=0.001$ the $\alpha$ 
We can see from Fig. 2that when $t \rightarrow T$,the geodesic and the real is unlikely seriously.It just because the self bug of the Tikhonov regularization method.

In fact,the consided problem (1) is converted to the problem described by (4).The inverse problem is to get $\omega(t)$ through $v(d, t)$ by the first Volteral equation

$K \omega(t)=\int_{0}^{t} \frac{\alpha e^{-\frac{(d-X(t))^{2}}{4 \alpha(t-\xi)}}}{2 \sqrt{\pi \alpha(t-\xi)}} \omega(\xi) d \xi=\int_{0}^{t} G^{\prime}(t, \xi) \omega(\xi) d \xi=f^{*}(t)$

From the equation above,easy to know that ,to confirm $\omega(t)$ on $[0, t)$ only need $f^{*}(t)$ on $[0, t)$, which just reflect the causality of the problem.that is,the knowledge of $f^{*}(t)$ on the past $[0, t)$ already reflects the value of $\omega(t)$ on $[t, T]$, and have nothing to do with the knowledge of $f^{*}(t)$ on $[t, T]$

However.,when used the Tiknonov regularization method on equation (12) to get $\omega(t)$,it is converted to the equation shown as (13).

$$
\int_{\xi}^{T} G^{\prime}(t, \xi) \int_{0}^{t} G^{\prime}(t, \tilde{\xi}) \omega(\tilde{\xi}) d \tilde{\xi} d t+\alpha \omega(t)=\int_{\xi_{i}}^{T} G^{\prime}\left(t, \xi_{i}\right) f^{\delta}(t) d t
$$

It is a posed second Fredholm equation, which is valid to get $\omega_{\partial}(t)$,the steady approximate solution of $\omega(t)$.

But,notice that the causality reflected by equation(12) is changed.seeing form (13),it converts to get $\omega(t)$ on $[0, t)$ by $f^{*}(t)$ on $[t, T]$,that is, when to get $\omega(t)$,the future value is used to get now value,which is out of reason.It is obvious that when $t \rightarrow T$,consider both sides of equation(13),the right is tended to zero,and the first part of the left is tended to zero.Then $\alpha \omega(t) \rightarrow 0$,that is $\omega(t) \rightarrow 0$.That is the reason of expressly glide when $t \rightarrow T$ on Figure2.

It is the self-bug of Tikhonov regularization method on special case.The Landweber method or the ductibility continuously method can be used to treate the case.Here we will use method of ductibility continuously to avoid the complexion.The result is shown in Figure3.

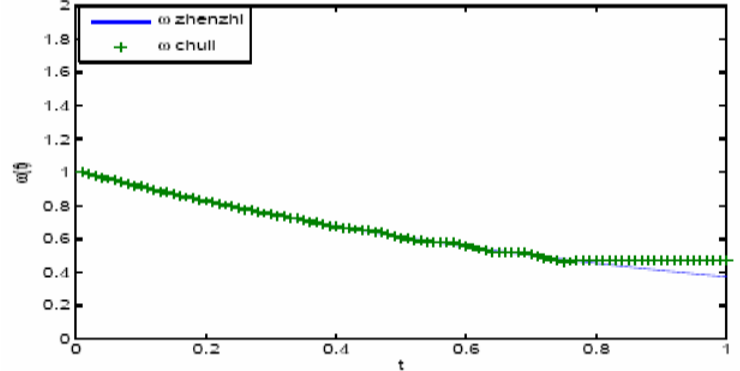

Figure 3. figure of $\omega(t)$ treated

Then using the (10),we can get $q(t)$.It's figure is as following Figure4

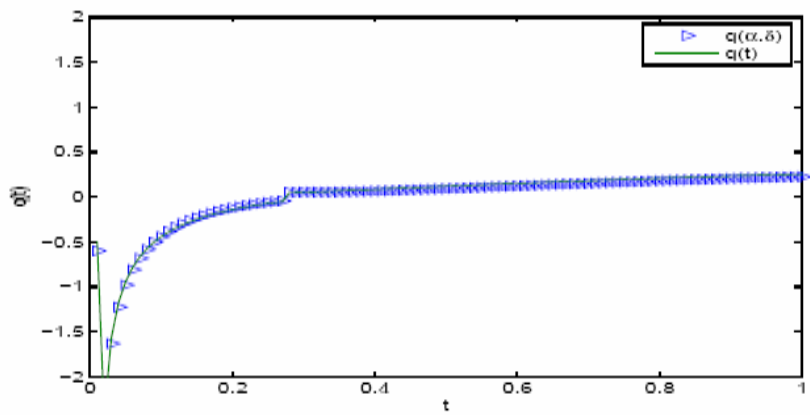

Figure 4. figure of $q(t)$

\section{REFERENCES}

[1] A Friedman, Differential Equations of Parabolic Type,Florida: Krieger(1983).

[2] DU Hua-dong. Studies of VariationaI Assimilation for the Inversion of the Coupled Air-sea Model.Manne Science Bulletin, 11, 13-22 ,(2009).

[3] Huang Sixun,A method of making up the satellite retrieval data of sea surface temperature , Scientia Meteorologica Sinica,28,237-43,(2008).

[4] Nersisyan H B and Osipyan D A. The moving boundary problem in the presence of a dipole magnetic field[J]. J. Phys. A: Math. Gen., 39, 7531$7542,(2006)$

[5] Qu Jian,Simulation Research of Point-sources Pollution Diffusion in the Jiaozhou Bay , Water Power,34,13-15,(2008).

[6] Pan Jishun. 2D multi-scale hybrid optimization method for geophysical inversion and its applica-tion. 6,337-348,(2009).

[7] Wang qiang,A Cartesian Mesh Algorithm for Supersonic Flows Around Arbitrary Moving Bodies,Chinese Journal of Computational Physics,24,517-526,(2009)

[8] J.J.Liu,Regularization methods of ill-posed problems and its applications(Published in China), Beijing,(2005).

[9] R Kress, Linear Integral Equations, Springer-Verlag, Berlin, (1989).

[10] Wang Haibo,Back Analysis and Calculation of Concrete Thermal Parameters Based on In-situ Test, Journal of China Three Gorges University(Natural Sciences) 31,33-35,(2009). 